\title{
A Distributed Network for Multimodal Experiential Representation of Concepts
}

2

23 Pages: 33

24 Figures: 4

25 Supplementary Tables: 2

26 Financial interests or conflicts of interest: None.

Abbreviated title: Multimodal Concept Representation

\section{Corresponding Author:}

Leonardo Fernandino, Ph.D.

Departments of Neurology and Biomedical Engineering Medical College of Wisconsin 8701 W Watertown Plank Rd, Milwaukee, WI 53226

Email: Ifernandino@mcw.edu

Office Phone: 414-955-7388
${ }^{1}$ Department of Neurology, Medical College of Wisconsin, Milwaukee, WI, USA

${ }^{2}$ Department of Biophysics, Medical College of Wisconsin, Milwaukee, WI, USA ${ }^{3}$ Department of Biomedical Engineering, Medical College of Wisconsin and Marquette University, Milwaukee, WI, USA

Key words: semantics, concept representation, representational similarity analysis, fMRI 
bioRxiv preprint doi: https://doi.org/10.1101/2021.07.05.451188; this version posted July 6, 2021. The copyright holder for this preprint (which

was not certified by peer review) is the author/funder, who has granted bioRxiv a license to display the preprint in perpetuity. It is made available under aCC-BY-NC-ND 4.0 International license.

\section{Acknowledgements:}

28 This work was supported by National Institute on Deafness and Other Communication Disorders

29 (NIDCD) grant R01 DC016622, by the Intelligence Advanced Research Projects Activity under

30 Grant FA8650-14-C-7357, and by a grant from the Advancing a Healthier Wisconsin Foundation

31 (Project \#5520462). The authors thank Volkan Arpinar, Elizabeth Awe, Joseph Heffernan,

32 Steven Jankowski, Jedidiah Mathis, and Megan LeDoux for technical assistance.

33

34

35

36

37

38

39

40

41

42

43

44 


\section{Abstract}

The architecture of the cortical system underlying concept representation is a topic of intense debate. Much evidence supports the claim that concept retrieval selectively engages sensory, motor, and other neural systems involved in the acquisition of the retrieved concept, yet there is also strong evidence for involvement of high-level, supramodal cortical regions. A fundamental question about the organization of this system is whether modality-specific information originating from sensory and motor areas is integrated across multiple "convergence zones" or

52 in a single centralized "hub". We used representational similarity analysis (RSA) of fMRI data to map brain regions where the similarity structure of neural patterns elicited by large sets of concepts matched the similarity structure predicted by a high-dimensional model of concept representation based on sensory, motor, affective, and other modal aspects of experience. Across two studies involving different sets of concepts, different participants, and different tasks, searchlight RSA revealed a distributed, bihemispheric network engaged in multimodal experiential representation, composed of high-level association cortex in anterior, lateral, and ventral temporal lobe; inferior parietal lobule; posterior cingulate gyrus and precuneus; and medial, dorsal, ventrolateral, and orbital prefrontal cortex. These regions closely resemble networks previously implicated in general semantic and "default mode" processing and are known to be high-level hubs for convergence of multimodal processing streams. Supplemented by an exploratory cluster analysis, these results indicate that the concept representation system consists of multiple, hierarchically organized convergence zones supporting multimodal integration of experiential information.

\section{Significance Statement}

68 It has long been known that information about visual, auditory, motor, affective, and other

69 features of our phenomenal experience originate in distinct brain regions. However, it is still 70 unclear how these processing streams converge to form multimodal concept representations. 
71 Using fMRI together with a multimodal experiential model of conceptual content, we show in two

72 large studies that concept knowledge is represented across a distributed, bihemispheric network

73 including temporal, parietal, limbic, and prefrontal association cortices. These results argue

74 against the idea of a single centralized "hub" for concept representation, suggesting instead that

75 multiple high-level convergence zones encode conceptual information in terms of multimodal

76 experiential content.

77

\section{Introduction}

Concepts are the building blocks of meaning and are essential for everyday thinking, planning, and communication, yet there remains considerable debate surrounding their neural implementation. "Grounded" theories of concept representation postulate that sensory-motor and affective representations involved in concept formation are re-activated during concept retrieval (Damasio, 1989; Barsalou, 2008; Glenberg et al., 2009). Support for this claim includes many studies showing that perceptual and motor processing areas are activated when corresponding perceptual or motor information about concepts is retrieved (Meteyard and Vigliocco, 2008; Binder and Desai, 2011; Kiefer and Pulvermuller, 2012; Kemmerer, 2014). How these multiple modality-specific representations are combined during concept retrieval, however, is not yet clear. Primate cortex contains multiple regions where information converges across sensory modalities (Jones and Powell, 1970; Mesulam, 1998; Man et al., 2013; Man et al., 2015). Portions of the human superior temporal sulcus (STS), for example, are known to respond to tactile, auditory, and visual stimulation (Beauchamp et al., 2008). The homolog of this region in macaque monkeys contains neurons that similarly respond to any of these stimulation modalities (Bruce et al., 1981) and are anatomically connected to corresponding unimodal cortex (Padberg et al., 2003). Other primate brain areas reported to have multimodal characteristics include posterior parietal cortex (Andersen, 1997), prefrontal cortex (Sugihara et al., 2006), parahippocampus (Damasio et al., 1982), and entorhinal cortex 
(Van Hoesen et al., 1972). Possible human homologs of these regions include cortical areas identified with the "default mode network", as suggested by a step-wise connectivity analysis of resting-state fMRI (Sepulcre et al., 2012). Beginning with seed regions in multiple primary sensory cortices, these authors showed that connections arising from these regions gradually converge, over multiple connectivity steps, at high-level "hubs" that include much of the lateral temporal cortex, angular gyrus, dorsomedial and inferolateral prefrontal cortex, posterior cingulate gyrus and precuneus.

Various models propose a central role for multimodal or supramodal hubs in concept processing, though both the anatomical location and information content encoded in these hubs remain unclear (Mahon and Caramazza, 2008; Binder and Desai, 2011; Lambon Ralph et al., 2017). One prominent theory proposes that the anterior temporal lobe (ATL) plays a unique role in storing abstract concept representations. During concept retrieval, the central ATL hub would activate modality-specific representations stored in unimodal cortical areas (the "spokes") (Patterson et al., 2007). An alternative model postulates widespread and hierarchically organized convergence zones in multiple brain locations (Damasio, 1989; Mesulam, 1998; Meyer and Damasio, 2009). We have previously proposed that these convergence zones are neurally implemented in the multimodal connectivity hubs identified by Sepulcre et al. (2012), which closely correspond to the regions identified in a large neuroimaging meta-analysis of semantic word processing (Binder et al., 2009). This idea is supported by neuroimaging findings indicating that these cortical regions encode multimodal information about the experiential content of lexical concepts (Bonner et al., 2013; Fernandino et al., 2016b; Fernandino et al., 2016a; Fernandino et al., 2021).

Here we use representational similarity analysis (RSA) with a whole-brain searchlight approach to identify cortical regions involved in multimodal conceptual representation. RSA measures the level of correspondence between the similarity matrix for a set of stimuli (e.g., words) derived from neural data and the similarity matrix for the same stimulus set computed 
from an a priori representational model (Kriegeskorte et al., 2008). We used a searchlight approach (Kriegeskorte et al., 2008) to generate a map of cortical regions where this representational correspondence holds true. We used an experiential model of conceptual content as the basis for RSA (Binder et al., 2016). Unlike the abstract representations used in previous RSA studies, this model encodes conceptual content explicitly in terms of 65 sensory, motor, affective, and other experiential processes. Identical analyses were run on two large datasets to assess replication across independent participant samples, word sets, and tasks. The analyses provided strong evidence that the multimodal experiential content of lexical concepts is represented across several high-level convergence zones.

\section{Material and Methods}

\section{Experiment 1}

Participants

Nineteen native English speakers (11 women, 8 men) participated in Experiment 1. Their mean age was 26.4 years (range 20 to 38 ). All were right-handed according to the Edinburgh handedness inventory (Oldfield, 1971) and had no history of neurological disease. All participants in Experiments 1 and 2 were compensated for their time and gave informed consent in conformity with a protocol approved by the Institutional Review Board of the Medical College of Wisconsin.

\section{Stimuli and Concept Features}

The stimuli consisted of 242 words, including 141 nouns, 62 verbs, and 39 adjectives (Supplementary Table 1). The noun concepts included inanimate objects, animate objects, human roles (e.g., mother, doctor), settings (e.g., church, forest), and events. Stimuli were selected by the Intelligence Advanced Research Projects Activity, which funded the study. Experiential representations for these words were available from a previous study in which 
ratings on 65 experiential domains were used to represent word meanings in a high-dimensional space (Binder et al., 2016). In brief, the experiential domains were selected based on known neural processing systems - such as color, shape, visual motion, touch, audition, motor control, olfaction - as well as other fundamental aspects of cognition whose neural substrates are less clearly understood, such as space, time, affect, reward, numerosity, and others. Ratings were collected using the crowd sourcing tool Amazon Mechanical Turk, in which volunteers rated the relevance of each experiential domain to a given concept on a 0-6 Likert scale. The value of each feature was represented by averaging ratings across participants. This feature set was highly effective at clustering concepts into traditional taxonomic categories (e.g., animals, plants, vehicles, occupations, etc.) (Binder et al., 2016) and has been used successfully to decode fMRI activation patterns during sentence reading (Anderson et al., 2017; Anderson et al., 2019).

\section{Stimulus Presentation and Tasks}

163 Words were presented visually in a fast event-related procedure with variable inter-stimulus order across two separate imaging sessions (3 presentations per session).

168 Participants were instructed to read each word silently and think about the meaning of the word.

169 To ensure attention to the stimuli, a random $10 \%$ of the trials were followed by a semantic-

170 matching probe task, in which 2 words were shown side by side, and the participant indicated by

171 a button press which of the two was more similar in meaning to the word just presented (these

172 probe trials were not included in the analyses). All trials then concluded with presentation of a

173 nonverbal "reset" stimulus for 1.5 seconds, the aim of which was to suppress processing of the

174 previously presented word. The reset stimulus consisted of two grey squares presented side by 
side and separated by a vertical black line. The participant indicated by a button press which of the two squares was brighter. A variable fixation period of $0-4 \mathrm{sec}$ followed all trials prior to the beginning of the next trial.

\section{"Think about the meaning of each word, and respond to a question about the word meaning on some trials."}

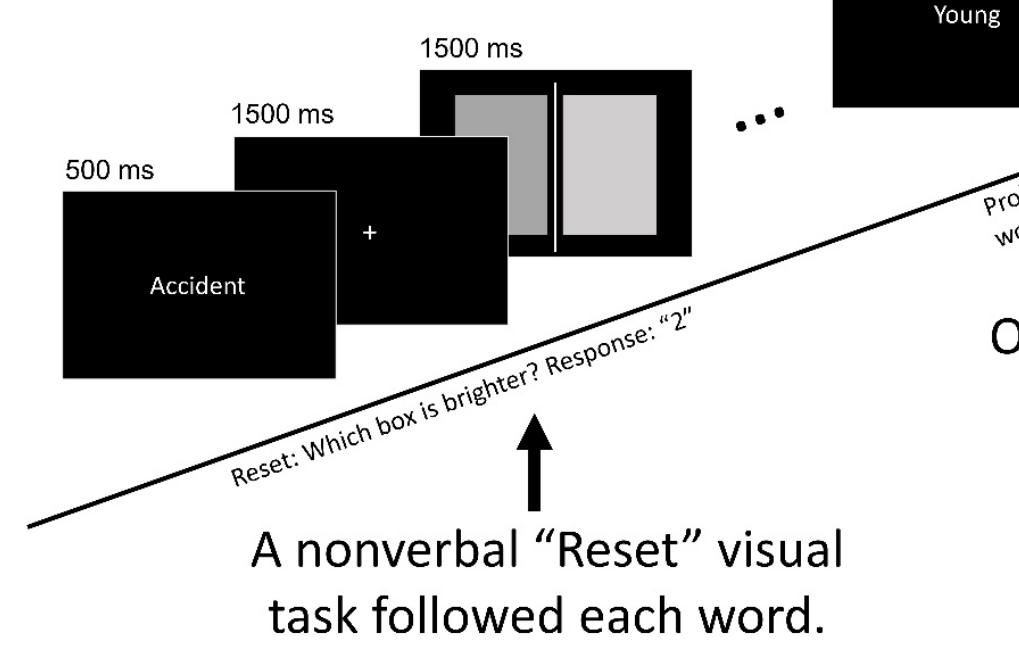

$2000 \mathrm{~ms}$

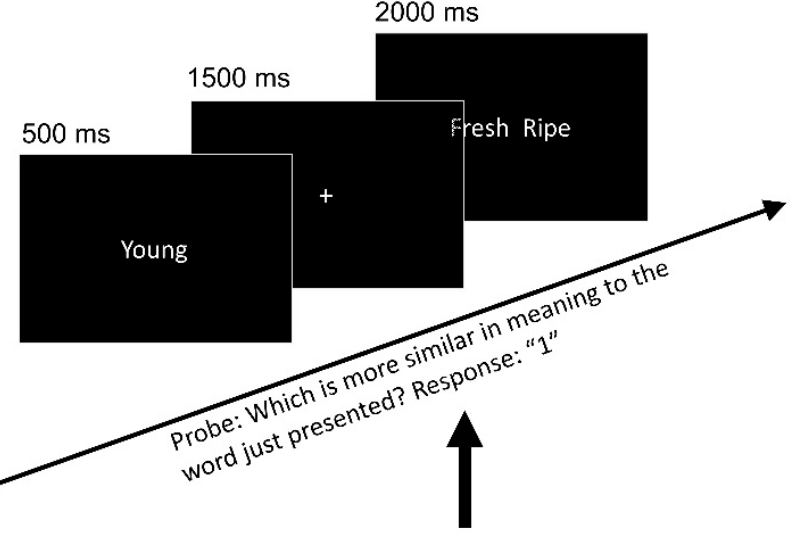

On a random $10 \%$ of trials, the stimulus word was followed by a semantic matching probe trial.

Figure 1. Schematic illustration of the tasks used in Experiment 1.

Each presentation of 242 test words and 26 probe trials ( 268 trials in total) occurred over the course of four imaging runs, each lasting 6 minutes. The four runs that comprised one repeat of the entire list was referred to as a "set". To minimize lexical ambiguity, grammatical class was used to block items by run. This was necessary because many of the nouns in the list can also be used as verbs, and several had very different semantic features when used as nouns vs. verbs (e.g., 'left', 'duck', 'saw', 'fence', 'spring'). Although the verbs in the set were all in past tense, several are also used commonly as adjectival participles ('celebrated', 'damaged', 'lost', 'planned', 'used'). Thus, nouns and verbs were presented in different runs, and adjectives 
were blocked with the nouns to separate them from the verbs. The nouns and adjectives were distributed evenly across 3 of the 4 imaging runs in each set, with the runs balanced on word class and noun category. Each of these 3 runs thus included 13 adjectives, 4-5 event nouns, 8 animate object nouns, 12-13 inanimate object nouns, 12-13 human role nouns, and 10 setting nouns. The remaining run of each set contained all 62 verbs.

Stimuli for probe trials were selected pseudo-randomly with replacement. For the 3 noun-adjective runs, a random set of either 6 or 7 probe trial words was selected for each run from the words comprising the other two noun-adjective runs, such that no stimulus used on a probe trial was repeated within the same run. This was not possible for the verb run, since all 62 verbs were presented in the same run. Thus, for the verb run, 7 verbs appeared twice - one time followed by a probe and one time without a probe.

Nine complete sets were composed in this way. Six were selected for each participant, with counterbalancing across participants. In addition, the order of presentation within each run was randomized for each participant to eliminate order effects at the group level.

\section{MRI Data Acquisition and Processing}

Images were acquired with a 3T GE 750 scanner at the Center for Imaging Research of the Medical College of Wisconsin. High-resolution T1-weighted anatomical images were acquired with a 3D spoiled gradient echo sequence $(F O V=240 \mathrm{~mm}, 220$ axial slices, in-plane matrix = $256 \times 224$, voxel size $=1 \times 1 \times 1 \mathrm{~mm}^{3}$ ). T2-weighted anatomical images were acquired with a CUBE T2 sequence $(F O V=256$ mm, 168 sagittal slices, in-plane matrix $=256 \times 256$, voxel size $=1 \times 1 \times 1 \mathrm{~mm}^{3}$ ). T2* -weighted gradient-echo echoplanar images were obtained for functional imaging $\left(\mathrm{TR}=2000 \mathrm{~ms}, \mathrm{TE}=24 \mathrm{~ms}\right.$, flip angle $=77^{\circ}, \mathrm{FOV}=192 \mathrm{~mm}, 41$ axial slices, in-plane matrix $=64 \times 64$, voxel size $=3 \times 3 \times 3 \mathrm{~mm}^{3}$ ).

Preprocessing was performed using AFNI. EPI images were corrected for slice timing. All images were then aligned to the $3^{\text {rd }}$ functional image in the series before aligning to the T1- 
weighted anatomical image. All voxels were normalized to have a mean of 100 and a range of 0 included the 6 repetitions of the word and excluded any probe task trials involving that word, resulting in 242 beta coefficient maps. Regressors of no interest included 12 degrees of

221 freedom of head motion, response time for the reset task, and response time for the probe task

222 trials. Individual word, reset, and probe task event regressors were convolved with a hemodynamic response function. A $t$ statistical map was generated for each word and these maps were subsequently used for the searchlight RSA.

\section{Surface-Based Searchlight Representational Similarity Analysis}

227 To optimize alignment between participants and to constrain the searchlight analysis to cortical grey matter, individual brain surface models were constructed from T1-weighted and T2weighted anatomical data using Freesurfer and the HCP pipeline (Glasser et al., 2013). The cortex ribbon was reconstructed in standard grayordinate space with 2-mm spaced vertices. We visually checked the quality of reconstructed surfaces before carrying out the analysis. pipeline to produce the final surfaces. Only cortical grey matter was included in the analysis.

RSA was carried out using custom Python and Matlab scripts. Searchlight RSA typically employs spherical volumes moved systematically through the brain or the cortical grey matter voxels. This method, however, does not exclude signals from white matter voxels that happen to

237 fall within the sphere, and which may contribute noise. Spherical volumes may also erroneously

238 combine non-contiguous cortical regions across sulci. Surface-based searchlight analysis

239 overcomes these shortcomings using circular 2-dimensional "patches" confined to contiguous

240 vertices on the cortical surface. At each vertex, a fast-marching algorithm was applied to create

241 a 5-mm radius patch around the seed vertex on the midthickness surface, resulting in a group of 
vertices comprising each patch. These vertices were then mapped one by one back to the native volume space of the participant to label voxels associated with the surface patch. To avoid partial-volume effects, we included only voxels that contained the entire middle $80 \%$ of the cortical ribbon at the mapped vertex location. Each surface vertex was thus associated with a group of voxels in native space (the searchlight ROI) for subsequent RSA.

Representational dissimilarity matrices (RDMs) were calculated for the semantic model

248 (the model RDM) and for each vertex-associated ROI (the neural RDM). Each entry in the neural RDM represented the correlation distance between fMRI responses evoked by two different words. Neural RDMs were computed for each of the 64,984 vertices. For the model

251 RDM, we calculated the cosine distances between each pair of words in the 65-dimensional experiential feature space. A word length RDM, created by taking the absolute difference in letter length between each word pair, was included as a covariate matrix of no interest. Pearson correlations between neural RDMs and the model RDM were computed controlling for the word length RDM, resulting in a partial correlation score map on the surface for each participant.

Finally, second level analysis was performed on the partial correlation score maps after

257 alignment of each individual map to a common surface template (generated by averaging the 258 individual 32k_FS_LR meshes produced by the HCP pipeline), Fisher z-transformation, and smoothing of the maps with a 6-mm full width at half maximum Gaussian kernel. A one-tailed, one-sample t-test against zero was applied at all vertices. FSL's PALM was used for nonparametric permutation testing to determine cluster-level statistical inference. Cluster-level

262 statistical inference was implemented with a cluster-forming threshold of $z>3.1(p<0.001)$.

263 The distribution of the largest clusters across permutations, in which the correlations were 264 randomly sign-flipped 10,000 times, was calculated, and a significance level of $\alpha<0.01$ was set. 265 The final data were rendered on the group averaged HCP template surface. 


\section{Experiment 2}

269

270

271

272

273

274

275

276

277

278

279

280

281

282

283

284

285

\section{Participants}

Experiment 2 involved 22 right-handed, native English speakers (11 women, 11 men; mean age 29.1; range 20 to 41). None of the participants took part in Experiment 1. This dataset was reported in a previous study (Fernandino et al., 2021).

\section{Stimuli}

Stimuli (see Supplementary Table 2) included 160 object nouns (40 each of animals, foods, tools, and vehicles) and 160 event nouns (40 each of social events, verbal events, non-verbal sound events, and negative events). Of the 320 concepts included in Experiment 2, 24 objects and 9 events were also used in Experiment 1. Concept ratings on the same 65 experiential domains used for the model in Experiment 1 were obtained for each concept using the same crowd-sourcing methods as in Experiment 1.

\section{Stimulus Presentation and Tasks}

As in Experiment 1, words were presented visually in a fast event-related procedure with variable inter-stimulus intervals, and the entire list was presented 6 times in random order over three imaging sessions performed on separate days.

On each trial, a noun was displayed in white font on a black background for $500 \mathrm{~ms}$, followed by a 2.5 -second blank screen. Each trial was followed by a central fixation cross with variable duration between 1 and $3 \mathrm{~s}$ (mean $=1.5 \mathrm{~s}$ ). Participants rated each noun according to how often they encountered the corresponding entity or event in their daily lives, on a scale from 1 ("rarely or never") to 3 ("often"). This familiarity judgment task was designed to encourage semantic processing of the word stimuli without emphasizing any particular semantic features or dimensions. Participants indicated their response by pressing one of three buttons on a response pad with their right hand. In contrast to Experiment 1, no reset task was used. 
Each presentation of the 320 test words occurred over the course of 4 imaging runs.

Each session consisted of 2 presentations of the full list (8 runs).

\section{MRI Data Acquisition and Processing}

Images were acquired with a 3T GE Premier scanner at the Medical College of Wisconsin.

Structural imaging included a T1-weighted MPRAGE volume (FOV = $256 \mathrm{~mm}, 222$ axial slices,

voxel size $=0.8 \times 0.8 \times 0.8 \mathrm{~mm}^{3}$ ) and a T2-weighted CUBE acquisition (FOV = $256 \mathrm{~mm}, 222$

sagittal slices, voxel size $\left.=0.8 \times 0.8 \times 0.8 \mathrm{~mm}^{3}\right) . \mathrm{T}^{*}$-weighted gradient-echo echoplanar images were obtained for functional imaging using a simultaneous multi-slice sequence (SMS factor $=$

4, $\mathrm{TR}=1500 \mathrm{~ms}, \mathrm{TE}=23 \mathrm{~ms}$, flip angle $=50^{\circ}, \mathrm{FOV}=208 \mathrm{~mm}, 72$ axial slices, in-plane matrix $=$

$104 \times 104$, voxel size $\left.=2 \times 2 \times 2 \mathrm{~mm}^{3}\right)$. A pair of T2-weighted spin echo echo-planar scans (5

runs 4 and 5, and after run 8, to provide estimates of EPI geometric distortion in the phase-

311 Experiment 1, each word was treated as a single regressor of interest and convolved with a

312 hemodynamic response function, resulting in 320 beta coefficient maps. Head motion vectors were again included as regressors of no interest. Response time z-score on each trial of the

314 familiarity judgment task was also included as a covariate of no interest.

\section{Surface-Based Searchlight Representational Similarity Analysis}

317 RSA analysis, generation of group maps, and thresholding methods for Experiment 2 were 318 identical to those used for Experiment 1. 


\section{Hierarchical clustering based on neural similarity structure}

321

322

Although the regions identified in Experiments 1 and 2 all show neural similarity structures that are correlated with the semantic model structure, it is possible that they vary somewhat in their information content. To investigate relative differences and similarities between the representational structure of the various regions identified in the RSAs, we performed hierarchical clustering analysis on the neural RDMs of these regions, as follows. First, the group maps from each experiment were thresholded at $p<.0005$ to separate minimally connected regions and highlight vertices with strong correlations to the semantic model. These maps were then overlapped to identify vertices common to both experiments. These steps resulted in 23 regions common to both analyses, which were used as regions of interest (ROIs) for the hierarchical clustering analysis. At the individual participant level, within each ROI, seed vertices with the highest correlation scores were combined iteratively until a set of approximately 100 voxels associated with these vertices was compiled. A neural RDM was then computed for each such voxel set, resulting in 23 RDMs for each individual. Pairwise RDM correlation was calculated for these 23 voxel sets at the individual level, resulting in a new $23 \times 23$ matrix in which each entry represented the correlation between neural RDMs of two ROls. These matrices were then averaged across all 41 participants, and hierarchical clustering was implemented on this averaged matrix, excluding the diagonal. Ward's variance minimization algorithm was applied to calculate distances between clusters.

\section{Results}

Experiment 1. "Probe" trials requiring semantic forced-choice matching of 2 words with the preceding list word were presented after $10 \%$ of list words to encourage attention to the list words. One participant failed to provide responses on this task, probably due to inadequate instruction. For the remaining participants, the mean response rate was $95.5 \%$ (SD $4.2 \%$ ), and mean accuracy was $82.1 \%$ (SD 7.0\%). A perceptual "reset" task occurred after all list words 
346 (and probe trials) with the aim of curtailing processing of the previous list word. The mean

347 response rate on this task was $98.9 \%$ (SD 2.5\%), and mean accuracy was $96.1 \%$ (SD 4.6\%).

Group-level searchlight RSA showed a bilateral, distributed network of regions where neural similarity correlated with semantic similarity across the 242 list words (Figure 2, left).

350 Extensive temporal lobe involvement included much of the temporal pole, superior temporal

351 sulcus (STS) and middle temporal gyrus (MTG), and anterior fusiform and parahippocampal gyri

352 bilaterally. The inferior temporal gyrus (ITG) was also involved, more so on the left. Parietal lobe

353 involvement was mainly in the inferior parietal lobule, including angular and supramarginal gyri

354 (AG and SMG) bilaterally. Frontal lobe regions included the inferior frontal gyrus (IFG), much of

355 the superior frontal gyrus (SFG) laterally and medially, more restricted patches in the middle

356 frontal gyrus, and orbital frontal cortex bilaterally. Small regions of the precentral gyrus were

357 involved in both hemispheres, and there was substantial involvement of the right insula. On the

358 medial surface there was extensive involvement of the posterior cingulate gyrus and adjacent

359 precuneus bilaterally, and the rostral anterior cingulate cortex bilaterally. 

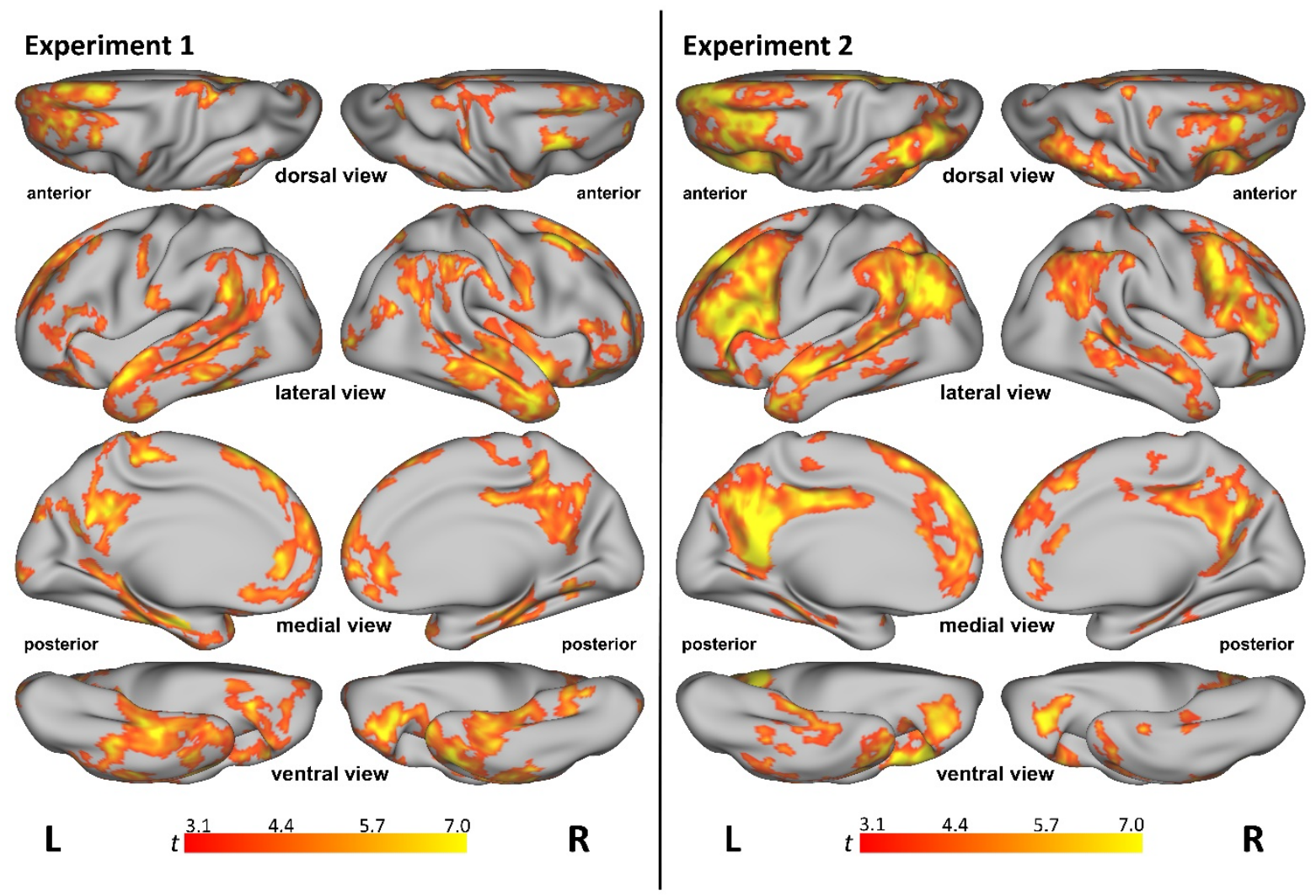

Figure 2. Brain areas where similarity between the neural patterns evoked by concepts was significantly correlated with concept similarity according to the semantic model. Results for Experiment 1 (left) and Experiment 2 (right) are shown on dorsal, lateral, medial, and ventral surface views. All results are significant at $p<0.001$ and cluster corrected at $\alpha<0.01$. Colors represent $t$ values.

Experiment 2. The mean response rate on the familiarity judgment task was $98.6 \%$ (SD $2.3 \%$ ).

375 Intra-individual consistency in familiarity ratings across the 6 repetitions of each word was

376 evaluated using intraclass correlations (ICCs) based on a single measurement, two-way mixed

377 effects model and the absolute agreement definition. Results suggested generally good overall

378 intra-individual agreement, with individual ICCs ranging from fair to excellent (mean ICC =

3790.661 , range: $0.438-0.858$, all $p s<0.00001)$ (Cicchetti, 1994). To examine consistency in 
380 familiarity ratings across participants, responses to the 6 repetitions were first averaged within

381 individuals, and the ICC across participants was calculated using the consistency definition.

382 The resulting ICC of 0.595 (95\% confidence interval $[0.556,0.635], p<.00001)$ suggested fair

383 to good inter-individual consistency.

384 As with Experiment 1, group-level searchlight RSA showed a bilateral, distributed

385 network of regions where neural similarity correlated with semantic similarity across the test

386 concepts (Figure 1, right). Most of these overlapped with those in Experiment 1, including

387 temporal pole, STS, MTG, AG, SMG, IFG, SFG, and posterior cingulate/precuneus (PCC)

388 bilaterally. Compared to Experiment 1, there was notably more extensive involvement of lateral

389 prefrontal cortex, including inferior and middle frontal gyri, bilaterally, and somewhat less

390 extensive ventral temporal lobe involvement. Areas of overlap between the two experiments are 391 shown in Figure 3. 

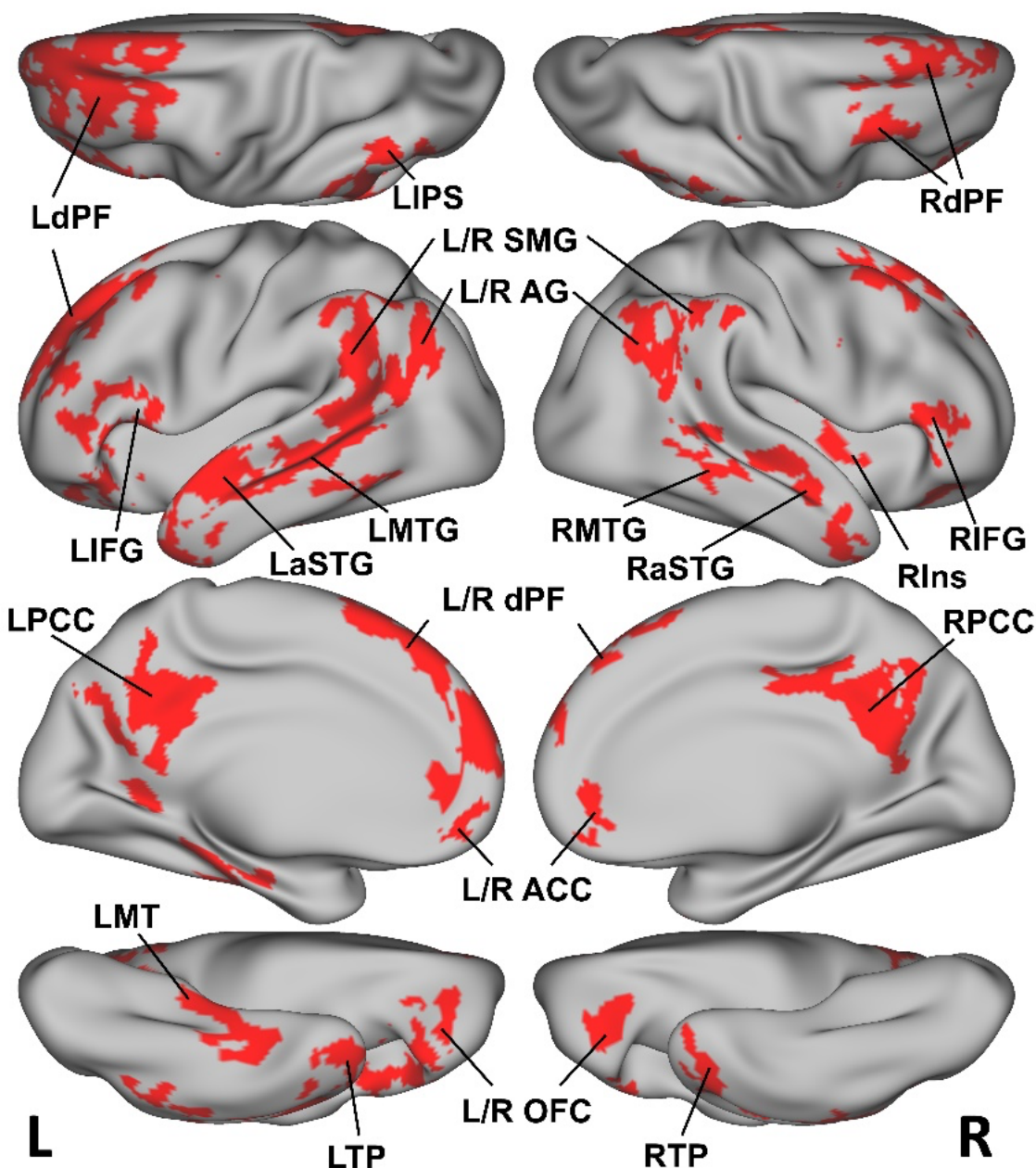

Figure 3. Brain areas where neural similarity was significantly correlated with model similarity in both Experiment 1 and Experiment 2. LACC: left anterior cingulate cortex; LAG: left angular gyrus; LaSTG: left anterior superior temporal gyrus; LdPF: left dorsal prefrontal cortex; LIFG: left inferior frontal gyrus; LIPS: left intraparietal sulcus; LMT: left medial temporal lobe; LMTG: left middle temporal gyrus; LOFC: left orbital frontal cortex; LPCC: left posterior cingulate and precuneus cortex; LSMG: left supramarginal gyrus; LTP: left temporal pole; RAG: right angular gyrus; RaSTG: right anterior superior temporal gyrus; RdPF: right dorsal prefrontal cortex; RIFG: right inferior frontal gyrus; RIns: right insula; RMT: right medial temporal lobe; RMTG: right middle temporal gyrus; ROFC: right orbital frontal cortex; RPCC: right posterior cingulate and precuneus cortex; RSMG: right supramarginal gyrus; RTP: right temporal pole. 
Hierarchical clustering of neural similarity structures. Overlap of the Experiment 1 and

Experiment 2 RSA maps showed 23 regions common to both (color-coded in Figure 4, right).

407 Degree of similarity between the neural RDMs extracted from each of these regions was

408 examined using hierarchical cluster analysis (Figure 4, left). The results revealed a division

409 between ROls in the parietal lobe (PCC, AG, SMG, left intraparietal sulcus) and lateral temporal

410 lobe in one major cluster, and ROIs in the medial temporal lobe, temporal pole, and frontal lobes

411 in another major cluster. The parietal/lateral temporal cluster was further divided by hemisphere,

412 such that right AG, SMG, MTG, and anterior STG fell in one subcluster, and left parietotemporal

413 ROls in another, along with left and right PCC. The other main cluster included a "limbic"

414 subcluster consisting of bilateral temporal poles, parahippocampus/hippocampus, and right

415 insula. A final subcluster included all frontal lobe ROls and the left anterior STG.
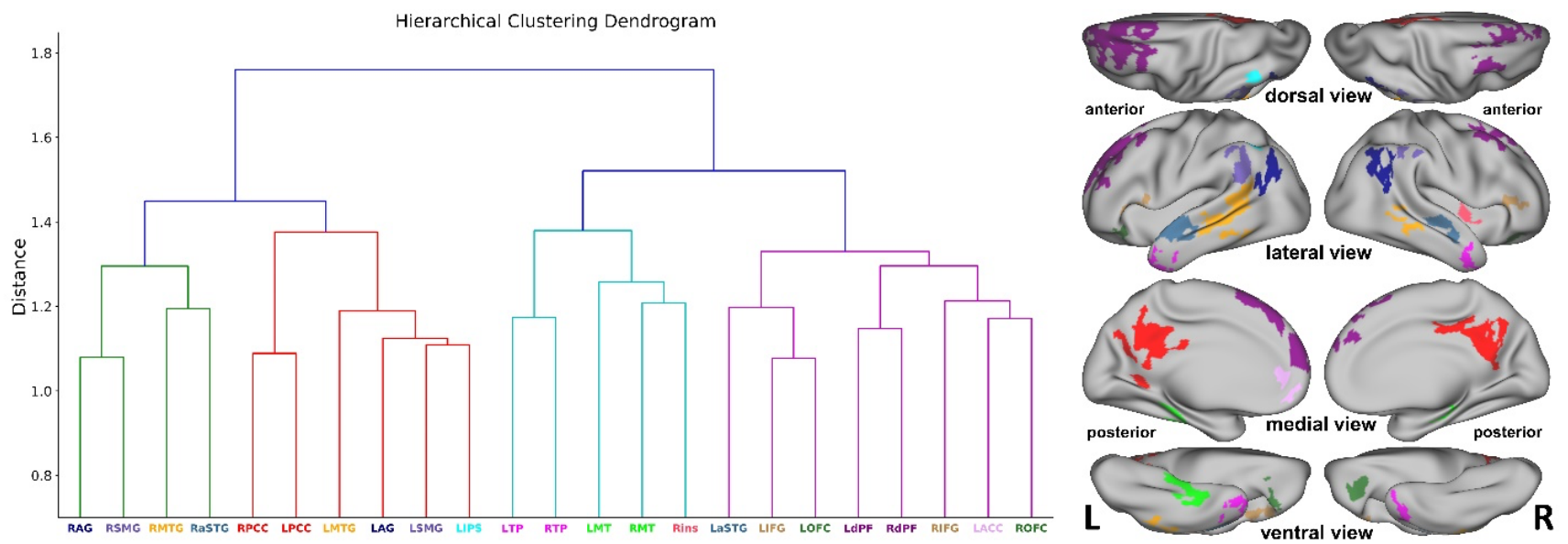

419 Figure 4. Results of hierarchical clustering of neural similarity structures. Left: Dendrogram

420 based on the averaged similarity structures of neural data from 23 ROls. The vertical axis

421 indicates linkage distance. Right: The 23 ROls defined by overlapping the RSA maps from the 
422 two experiments after thresholding each map at $p<0.0005$ and cluster-correcting at $\alpha<0.01$.

423 Anatomical labels match those in Figure 3.

\section{Discussion}

426 We sought to clarify the large-scale architecture of the concept representation system by

427 identifying cortical regions whose activation patterns encoded multimodal experiential

428 information about individual lexical concepts. Previous whole-brain searchlight RSA studies on

429 this topic have used semantic representation models based on category membership, semantic feature lists, or word co-occurrence statistics, producing highly variable results. Here we used a model based entirely on multimodal experiential content with no explicit reference to taxonomic or distributional similarity. Across two independent experiments, each involving a large number and a wide range of concepts, we detected multimodal concept representation in widespread heteromodal cortical regions, bilaterally, including anterior and posterior temporal cortex, inferior parietal cortex, posterior cingulate gyrus and precuneus, and medial, dorsal, ventrolateral, and ventral prefrontal regions. These results call into question the idea that information streams originating in unimodal cortical areas are integrated at a single anatomically localized hub for concept representation.

Four previous RSA studies using semantic models and word stimuli implicated anteromedial temporal cortex, particularly perirhinal cortex, as a semantic hub (Devereux et al.,

441 2013; Liuzzi et al., 2015; Martin et al., 2018; Liuzzi et al., 2019). All used semantic models

442 based on crowd-sourced feature production lists, and all used a feature verification task during

443 fMRI (e.g., "WASP - Does it have paws?"). Validity issues with feature production lists have 444 been noted previously, such as the fact that many features people produce are multimodal or 445 highly abstract, and some types of features are difficult to verbalize or systematically ignored 446 (Hoffman and Lambon Ralph, 2013). Another potential problem with these RSA studies is that 447 the verification task used during $\mathrm{fMRI}$ requires semantic processing of the explicitly named 
feature, which logically must contribute to the observed neural activation pattern but is not coded in the semantic model. These problems may have weakened the ability of these studies to detect other regions involved in concept representation.

Prior studies combining searchlight RSA with either taxonomic (Devereux et al., 2013; models have implicated more widespread regions, including posterior lateral temporal cortex,

454 inferior parietal lobe, posterior cingulate gyrus, and prefrontal cortex. Only one of these studies

455 (Anderson et al., 2015) reported any representational correspondence in medial or ventral

456 temporal areas. The two studies using taxonomic models (Devereux et al., 2013; Carota et al.,

457 2021) showed similar involvement of the left posterior superior temporal sulcus and MTG, with extension into adjacent AG and SMG. In contrast, the two studies using distributional models

459 (Anderson et al., 2015; Carota et al., 2021) found little or no posterior temporal involvement, and inferior parietal involvement was confined mainly to the left SMG. Frontal cortex involvement was uniformly present but highly variable in extent and location across the studies. Two studies reported involvement of the posterior cingulate/precuneus (Devereux et al., 2013; Anderson et al., 2015).

Several factors may have negatively impacted sensitivity and reliability in these studies.

465 First, ROI-based RSAs show that, relative to experiential models of concept representation, 466 taxonomic and distributional models are consistently less sensitive to the neural similarity 467 structure of lexical concepts (Fernandino et al., 2021). Furthermore, most of the prior studies 468 used volume-based spherical searchlights, which typically sample a mix of grey and white 469 matter voxels, while the surface-based approach used in the present study ensures that only 470 contiguous cortical gray matter voxels are included, thus reducing noise from uninformative 471 voxels. Finally, the nature of the task and the particularities of the concept set used as stimuli 472 can affect both the sensitivity of the analysis and the cortical distribution of the RSA searchlight 473 map, and variations in these properties may underlie some of the variation in results across 
studies. We dealt with this issue by (1) employing large numbers of concepts from diverse semantic categories and (2) by analyzing data from two independent experiments to identify areas displaying reliable representational correspondence with the semantic model across different concept sets and different tasks.

The network of brain regions identified in the current study closely resembles the network identified previously in a meta-analysis of 120 functional imaging studies on semantic processing (Binder et al., 2009). The results provide novel evidence that these brain regions, consisting essentially of heteromodal association areas distant from primary sensory and motor systems, represent conceptual information in terms of multimodal experiential content. In contrast to previous RSA studies of concept representation (Devereux et al., 2013; Anderson et al., 2015; Martin et al., 2018; Carota et al., 2021), the network includes extensive cortex in the ATL, a region strongly implicated in high-level semantic representation (Lambon Ralph et al., 2017). Although the current results support a role for the ATL in concept representation, they argue against it having a unique role as a central integration hub.

The concept representation network identified in the current study also closely resembles the set of brain regions referred to as the "default mode network" (Buckner et al., 2008). This overlap supports the view that concept retrieval and manipulation are major components of the brain's "default mode" of processing (Binder et al., 1999; Binder et al., 2009; Andrews-Hanna et al., 2014). Our results add to prior evidence by showing that the representational structure of neural activity in these regions reflects the experiential content of lexical concepts.

The finding of extensive frontal lobe involvement in concept "representation" deserves comment. Studies of brain damaged individuals and functional imaging experiments in the healthy brain have long been interpreted as supporting the classic view that frontal cortex plays an operational control rather than an information storage function in the brain (Stuss and Benson, 1986; Kimberg and Farah, 1993; Thompson-Schill et al., 1997; Wagner et al., 2001). 
Nevertheless, nearly all RSA studies of concept representation have observed similarity structure correlations in prefrontal regions. Why would activity in these areas reflect semantic content? We believe these observations can be reconciled with the classic view by postulating a more fine-grained organization of control systems in the frontal lobe than is usually assumed in semantic theories. Rather than being composed of large, homogeneous areas with a nonspecific control function, control systems in the prefrontal cortex may be tuned, at a relatively small scale, to particular sensory-motor and affective features. Neurophysiological studies in nonhuman primates provide evidence for tuning of prefrontal neurons to preferred stimulus modalities (Romanski, 2007), as well as differential connectivity across the prefrontal cortex with various sensory systems (Barbas and Mesulam, 1981; Petrides, 2005). A few human functional imaging studies provide similar evidence for sensory modality tuning in prefrontal cortex (Greenberg et al., 2010; Michalka et al., 2015; Tobyne et al., 2017). If conceptual representation in temporal and parietal cortex is inherently organized according to experiential content, it seems plausible that controlled activation and short-term maintenance of this information would require similarly fine-grained control mechanisms.

This hypothesis finds some support in a comparison of our two experiments. The infrequent probe task procedure used in Experiment 1 was intentionally designed to minimize explicit, goal-directed retrieval of semantic information, and the inclusion of a non-semantic perceptual discrimination task after each trial likely encouraged participants to focus their attention on this task rather than on semantic retrieval. In contrast, the task in Experiment 2 required participants to make a semantic decision about each word. Compared to Experiment 1, the Experiment 2 results show much more extensive involvement of lateral prefrontal cortex. A likely interpretation is that the explicit task in Experiment 2 led to stronger engagement of feature-specific control networks in these frontal regions. We propose that the information represented in these prefrontal regions reflects their entrainment to experiential representations 

content. activation.

stored primarily in temporoparietal cortex, providing context-dependent control over their level of

Related to this issue is the question of how similar the many regions identified by RSA are to each other in terms of their representational structure. Although RSA ensures that the neural similarity structure of all these regions is related to the similarity structure encoded in the semantic model, representational structure should be expected to vary to some degree across distinct functional regions. An exploratory cluster analysis of the neural RDMs from these regions suggests a broad distinction between two clusters, one consisting of medial and lateral parietal cortex and posterior lateral temporal areas (across both hemispheres) and the other consisting of medial, ventral, and anterior temporal areas, right insula, and frontal areas. There was also evidence for a distinction between left and right parietotemporal representational structures. These results are consistent with proposed distinctions between the functions of frontal, posterior association, and limbic cortices, as well as longstanding claims regarding interhemispheric differences in semantic representation (Beeman and Chiarello, 1998). Interestingly, this analysis suggests that representations in the ATL are more similar to those in prefrontal areas than to those in posterior temporal and parietal areas involved in the representation of objects and events (Martin, 2007; Bedny et al., 2014). More research is needed to understand the factors that underlie these regional differences in representational 
545

546

547

548

549

550

551

552

553

554

555

556

557

558

559

560

561

562

563

564

565

566

567

\section{References}

Andersen RA (1997) Multimodal integration for the representation of space in the posterior parietal cortex. Philos Trans R Soc Lond B Biol Sci 352:1421-1428.

Anderson AJ, Bruni E, Lopopolo A, Poesio M, Baroni M (2015) Reading visually embodied meaning from the brain: Visually grounded computational models decode visual-object mental imagery induced by written text. Neuroimage 120:309-322.

Anderson AJ, Binder JR, Fernandino L, Humphries CJ, Conant LL, Raizada RDS, Lin F, Lalor EC (2019) An integrated neural decoder of linguistic and experiential meaning. J Neurosci 39:8969-8987.

Anderson AJ, Binder JR, Fernandino L, Humphries CJ, Conant LL, Aguilar M, Wang X, Doko D, Raizada RDS (2017) Predicting neural activity patterns associated with sentences using a neurobiologically motivated model of semantic representation. Cereb Cortex 27:4379-4395.

Andrews-Hanna JR, Smallwood J, Spreng RN (2014) The default network and self-generated thought: Component processes, dynamic control, and clinical relevance. Ann N Y Acad Sci 1316:29-52.

Barbas H, Mesulam MM (1981) Organization of afferent input to subdivisions of area 8 in the rhesus monkey. J Comp Neurol 200:407-431.

Barsalou LW (2008) Grounded cognition. Annu Rev Psychol 59:617-645.

Beauchamp MS, Yasar NE, Frye RE, Ro T (2008) Touch, sound and vision in human superior temporal sulcus. Neuroimage 41:1011-1020.

Bedny M, Dravida S, Saxe R (2014) Shindigs, brunches, and rodeos: The neural basis of event words. Cogn Affect Behav Neurosci 14:891-901.

Beeman M, Chiarello C (1998) Right hemisphere language comprehension: Perspectives from cognitive neuroscience: Psychology Press.

Binder JR, Desai RH (2011) The neurobiology of semantic memory. Trends Cogn Sci 15:527-536. 
Binder JR, Desai RH, Graves WW, Conant LL (2009) Where is the semantic system? A critical review and meta-analysis of 120 functional neuroimaging studies. Cereb Cortex 19:2767-2796.

Binder JR, Frost JA, Hammeke TA, Bellgowan PS, Rao SM, Cox RW (1999) Conceptual processing during the conscious resting state. A functional MRI study. J Cogn Neurosci 11:80-95.

Binder JR, Conant LL, Humphries CJ, Fernandino L, Simons SB, Aguilar M, Desai RH (2016) Toward a brain-based componential semantic representation. Cogn Neuropsychol 33:130-174.

Bonner MF, Peelle JE, Cook PA, Grossman M (2013) Heteromodal conceptual processing in the angular gyrus. Neuroimage 71:175-186.

Bruce C, Desimone R, Gross CG (1981) Visual properties of neurons in a polysensory area in superior temporal sulcus of the macaque. J Neurophysiol 46:369-384.

Buckner RL, Andrews-Hanna JR, Schacter DL (2008) The brain's default network: Anatomy, function, and relevance to disease. Ann N Y Acad Sci 1124:1-38.

Carota F, Nili H, Pulvermuller F, Kriegeskorte N (2021) Distinct fronto-temporal substrates of distributional and taxonomic similarity among words: Evidence from rsa of bold signals. Neuroimage 224:117408.

Damasio AR (1989) Time-locked multiregional retroactivation: A systems-level proposal for the neural substrates of recall and recognition. Cognition 33:25-62.

Damasio AR, Damasio H, Van Hoesen GW (1982) Prosopagnosia: Anatomic basis and behavioral mechanisms. Neurology 32:331-341.

Devereux BJ, Clarke A, Marouchos A, Tyler LK (2013) Representational similarity analysis reveals commonalities and differences in the semantic processing of words and objects. J Neurosci 33:18906-18916.

Fernandino L, Conant LL, Humphries CJ, Binder JR (2021) Decoding the information structure underlying the neural representation of concepts. bioRxiv:2021.2003.2016.435524. 
592

593

594

595

596

597

598

599

600

601

602

603

604

605

606

607

608

609

610

611

612

613

614

Fernandino L, Humphries CJ, Conant LL, Seidenberg MS, Binder JR (2016a) Heteromodal cortical areas encode sensory-motor features of word meaning. J Neurosci 36:9763-9769.

Fernandino L, Binder JR, Desai RH, Pendl SL, Humphries CJ, Gross WL, Conant LL, Seidenberg MS (2016b) Concept representation reflects multimodal abstraction: A framework for embodied semantics. Cereb Cortex 26:2018-2034.

Glasser MF, Sotiropoulos SN, Wilson JA, Coalson TS, Fischl B, Andersson JL, Xu J, Jbabdi S, Webster M, Polimeni JR, Van Essen DC, Jenkinson M, Consortium WU-MH (2013) The minimal preprocessing pipelines for the human connectome project. Neuroimage 80:105-124.

Glenberg AM, Webster BJ, Mouilso E, Havas D, Lindeman LM (2009) Gender, emotion, and the embodiment of language comprehension. 1:151-161.

Greenberg AS, Esterman M, Wilson D, Serences JT, Yantis S (2010) Control of spatial and feature-based attention in frontoparietal cortex. J Neurosci 30:14330-14339.

Hoffman P, Lambon Ralph MA (2013) Shapes, scents and sounds: Quantifying the full multi-sensory basis of conceptual knowledge. Neuropsychologia 51:14-25.

Jones EG, Powell TP (1970) An anatomical study of converging sensory pathways within the cerebral cortex of the monkey. Brain 93:793-820.

Kemmerer DL (2014) Cognitive neuroscience of language. New York, NY: Psychology Press.

Kiefer M, Pulvermuller F (2012) Conceptual representations in mind and brain: Theoretical developments, current evidence and future directions. Cortex 48:805-825.

Kimberg DY, Farah MJ (1993) A unified account of cognitive impairments following frontal lobe damage:

The role of working memory in complex, organized behavior. J Exp Psychol Gen 122:411-428.

Kriegeskorte N, Mur M, Bandettini P (2008) Representational similarity analysis - connecting the branches of systems neuroscience. Front Syst Neurosci 2:4. 
615

616

617

618

619

620

621

622

623

624

625

626

627

628

629

630

631

632

633

634

635

636

637

638

Lambon Ralph MA, Jefferies E, Patterson K, Rogers TT (2017) The neural and computational bases of semantic cognition. Nat Rev Neurosci 18:42-55.

Liuzzi AG, Dupont P, Peeters R, Bruffaerts R, De Deyne S, Storms G, Vandenberghe R (2019) Left perirhinal cortex codes for semantic similarity between written words defined from cued word association. Neuroimage 191:127-139.

Liuzzi AG, Bruffaerts R, Dupont P, Adamczuk K, Peeters R, De Deyne S, Storms G, Vandenberghe R (2015) Left perirhinal cortex codes for similarity in meaning between written words: Comparison with auditory word input. Neuropsychologia 76:4-16.

Mahon BZ, Caramazza A (2008) A critical look at the embodied cognition hypothesis and a new proposal for grounding conceptual content. J Physiol Paris 102:59-70.

Man K, Kaplan J, Damasio H, Damasio A (2013) Neural convergence and divergence in the mammalian cerebral cortex: From experimental neuroanatomy to functional neuroimaging. J Comp Neurol 521:4097-4111.

Man K, Damasio A, Meyer K, Kaplan JT (2015) Convergent and invariant object representations for sight, sound, and touch. Hum Brain Mapp 36:3629-3640.

Martin A (2007) The representation of object concepts in the brain. Annu Rev Psychol 58:25-45.

Martin CB, Douglas D, Newsome RN, Man LL, Barense MD (2018) Integrative and distinctive coding of visual and conceptual object features in the ventral visual stream. Elife 7.

Mesulam MM (1998) From sensation to cognition. Brain 121 ( Pt 6):1013-1052.

Meteyard L, Vigliocco G (2008) 15 - the role of sensory and motor information in semantic representation: A review. In: Handbook of cognitive science (Calvo P, Gomila A, eds), pp 291312. San Diego: Elsevier.

Meyer K, Damasio A (2009) Convergence and divergence in a neural architecture for recognition and memory. Trends Neurosci 32:376-382. 
Michalka SW, Kong L, Rosen ML, Shinn-Cunningham BG, Somers DC (2015) Short-term memory for space and time flexibly recruit complementary sensory-biased frontal lobe attention networks. Neuron 87:882-892.

Oldfield RC (1971) The assessment and analysis of handedness: The edinburgh inventory. Neuropsychologia 9:97-113.

Padberg J, Seltzer B, Cusick CG (2003) Architectonics and cortical connections of the upper bank of the superior temporal sulcus in the rhesus monkey: An analysis in the tangential plane. J Comp Neurol 467:418-434.

Patterson K, Nestor PJ, Rogers TT (2007) Where do you know what you know? The representation of semantic knowledge in the human brain. Nat Rev Neurosci 8:976-987.

Petrides M (2005) Lateral prefrontal cortex: Architectonic and functional organization. Philos Trans R Soc Lond B Biol Sci 360:781-795.

Romanski LM (2007) Representation and integration of auditory and visual stimuli in the primate ventral lateral prefrontal cortex. Cereb Cortex 17 Suppl 1:i61-69.

Sepulcre J, Sabuncu MR, Yeo TB, Liu H, Johnson KA (2012) Stepwise connectivity of the modal cortex reveals the multimodal organization of the human brain. J Neurosci 32:10649-10661.

Stuss DT, Benson DF (1986) The frontal lobes. New York: Raven Press.

Sugihara T, Diltz MD, Averbeck BB, Romanski LM (2006) Integration of auditory and visual communication information in the primate ventrolateral prefrontal cortex. J Neurosci 26:1113811147.

Thompson-Schill SL, D'Esposito M, Aguirre GK, Farah MJ (1997) Role of left inferior prefrontal cortex in retrieval of semantic knowledge: A reevaluation. Proc Natl Acad Sci U S A 94:14792-14797.

Tobyne SM, Osher DE, Michalka SW, Somers DC (2017) Sensory-biased attention networks in human lateral frontal cortex revealed by intrinsic functional connectivity. Neuroimage 162:362-372. 
bioRxiv preprint doi: https://doi.org/10.1101/2021.07.05.451188; this version posted July 6, 2021. The copyright holder for this preprint (which

was not certified by peer review) is the author/funder, who has granted bioRxiv a license to display the preprint in perpetuity. It is made available under aCC-BY-NC-ND 4.0 International license.

663 Van Hoesen GW, Pandya DN, Butters N (1972) Cortical afferents to the entorhinal cortex of the rhesus

664 monkey. Science 175:1471-1473.

665 Wagner AD, Pare-Blagoev EJ, Clark J, Poldrack RA (2001) Recovering meaning: Left prefrontal cortex

666 guides controlled semantic retrieval. Neuron 31:329-338.

667 


\section{Figure Legends}

Figure 1. Schematic illustration of the tasks used in Experiment 1.

Figure 2. Brain areas where similarity between the neural patterns evoked by concepts was significantly correlated with concept similarity according to the semantic model. Results for Experiment 1 (left) and Experiment 2 (right) are shown on dorsal, lateral, medial, and ventral surface views. All results are significant at $p<0.001$ and cluster corrected at $\alpha<0.01$. Colors represent $t$ values.

Figure 3. Brain areas where neural similarity was significantly correlated with model similarity in both Experiment 1 and Experiment 2. LACC: left anterior cingulate cortex; LAG: left angular gyrus; LaSTG: left anterior superior temporal gyrus; LdPF: left dorsal prefrontal cortex; LIFG: left inferior frontal gyrus; LIPS: left intraparietal sulcus; LMT: left medial temporal lobe; LMTG: left middle temporal gyrus; LOFC: left orbital frontal cortex; LPCC: left posterior cingulate and precuneus cortex; LSMG: left supramarginal gyrus; LTP: left temporal pole; RAG: right angular gyrus; RaSTG: right anterior superior temporal gyrus; RdPF: right dorsal prefrontal cortex; RIFG: right inferior frontal gyrus; RIns: right insula; RMT: right medial temporal lobe; RMTG: right middle temporal gyrus; ROFC: right orbital frontal cortex; RPCC: right posterior cingulate and precuneus cortex; RSMG: right supramarginal gyrus; RTP: right temporal pole.

Figure 4. Results of hierarchical clustering of neural similarity structures. Left: Dendrogram based on the averaged similarity structures of neural data from 23 ROls. The vertical axis indicates linkage distance. Right: The 23 ROIs defined by overlapping the RSA maps from the two experiments after thresholding each map at $p<0.0005$ and cluster-correcting at $\alpha<0.01$. Anatomical labels match those in Figure 3. 


\section{Supplementary Materials}

\begin{tabular}{|c|c|c|c|c|c|c|c|c|}
\hline \multicolumn{2}{|l|}{ Adjective } & \multicolumn{2}{|l|}{ Verb } & \multicolumn{5}{|l|}{ Noun } \\
\hline aggressive & soft & approached & left & accident & coffee & flower & office & team \\
\hline angry & spiritual & arrested & liked & activist & commander & football & parent & television \\
\hline big & tired & ate & listened & actor & company & forest & park & terrorist \\
\hline black & used & blocked & lived & agreement & computer & girl & party & theater \\
\hline blue & wealthy & bought & lost & airport & corn & glass & patient & ticket \\
\hline clever & white & broke & marched & army & council & guard & pencil & tourist \\
\hline cold & yellow & built & met & artist & couple & hall & pilot & tree \\
\hline dangerous & young & carried & negotiated & author & court & highway & plane & trial \\
\hline dark & & celebrated & opened & ball & criminal & horse & policeman & vacation \\
\hline dead & & crossed & planned & banker & debate & hospital & politician & victim \\
\hline dusty & & damaged & played & baseball & desk & hotel & priest & voter \\
\hline empty & & delivered & put & beach & dime & hurricane & prison & water \\
\hline expensive & & destroyed & ran & bed & dinner & island & protest & window \\
\hline famous & & drank & read & bicycle & diplomat & journalist & reporter & winter \\
\hline friendly & & drew & saw & bird & doctor & judge & restaurant & witness \\
\hline green & & dropped & shouted & boat & dog & jury & river & woman \\
\hline happy & & ended & slept & book & door & lab & school & worker \\
\hline heavy & & feared & spoke & boy & driver & lake & scientist & \\
\hline hot & & fed & stayed & bread & duck & lawyer & soccer & \\
\hline injured & & fixed & stole & bridge & editor & magazine & soldier & \\
\hline lonely & & flew & survived & businessman & egg & man & spring & \\
\hline long & & found & threw & cabinet & election & mayor & stone & \\
\hline loud & & gave & took & camera & embassy & medicine & store & \\
\hline new & & grew & used & car & engineer & meeting & storm & \\
\hline old & & held & visited & cash & family & minister & street & \\
\hline peaceful & & helped & walked & cellphone & farmer & mob & student & \\
\hline powerful & & hiked & wanted & chair & feather & morning & summer & \\
\hline red & & interviewed & watched & chicken & fence & mountain & sun & \\
\hline shiny & & kicked & went & child & field & mouse & table & \\
\hline sick & & landed & worked & church & fish & newspaper & tea & \\
\hline small & & laughed & wrote & cloud & flood & night & teacher & \\
\hline
\end{tabular}




\begin{tabular}{|c|c|c|c|c|c|c|c|}
\hline \multicolumn{4}{|l|}{ Event } & \multicolumn{4}{|l|}{ Object } \\
\hline Negative & Nonverbal & Social & Verbal & Animal & Food/Plant & Tool & Vehicle \\
\hline avalanche & chuckle & bash & lecture & hippopotamus & chestnut & scissors & ferry \\
\hline blizzard & screaming & conference & tribute & ant & cranberry & candle & train \\
\hline bombing & sizzle & rally & deposition & goldfish & flower & stapler & automobile \\
\hline downpour & siren & gathering & compliment & jackal & sauerkraut & umbrella & skateboard \\
\hline drought & crescendo & musical & greeting & cricket & mushroom & axe & carriage \\
\hline earthquake & snap & cruise & testimony & butterfly & pudding & crutches & helicopter \\
\hline epidemic & growling & wedding & discourse & mosquito & ham & comb & barge \\
\hline gunshot & shrieking & luncheon & dispute & alligator & chocolate & sandpaper & motorcycle \\
\hline gust & sobbing & outing & comment & trout & banana & book & jeep \\
\hline hail & clattering & jubilee & commemoration & chimpanzee & cider & faucet & van \\
\hline hailstorm & gulp & expo & denial & chicken & broccoli & pencil & bus \\
\hline hurricane & whine & reception & trial & duck & pumpkin & glass & plane \\
\hline inferno & whimpering & banquet & huddle & baboon & bread & hoe & tractor \\
\hline invasion & melody & reunion & advice & lion & cheese & fork & steamer \\
\hline landslide & hiccup & dance & quarrel & mouse & champagne & camera & car \\
\hline lightning & murmuring & feast & thanks & caterpillar & spaghetti & binoculars & rowboat \\
\hline monsoon & roaring & safari & interrogation & hawk & eggplant & straw & taxi \\
\hline squall & reverberation & contest & sermon & penguin & tobacco & football & canoe \\
\hline stampede & crackle & carnival & rant & whale & bean & microscope & locomotive \\
\hline storm & rustle & festival & lesson & crow & jam & hammer & trolley \\
\hline tempest & thunderclap & housewarming & threat & turkey & blueberry & thermometer & bicycle \\
\hline thunderstorm & jingle & fiesta & wisecrack & elephant & asparagus & baseball & sleigh \\
\hline tornado & clapping & march & grievance & bison & nectarine & keyboard & subway \\
\hline twister & chattering & christening & complaint & cheetah & coffee & key & escalator \\
\hline volcano & bellowing & prom & apology & rhinoceros & beer & ticket & sailboat \\
\hline war & grunt & cocktails & commentary & chipmunk & cherry & newspaper & elevator \\
\hline whirlwind & laughter & picnic & meeting & monkey & plant & hairbrush & submarine \\
\hline wildfire & groaning & tour & squabble & dolphin & carrot & skillet & scooter \\
\hline
\end{tabular}

